13

\title{
Зависимость наклона текстуры и эффективности возбуждения сдвиговых волн пленками ZnO от давления рабочего газа в магнетронной системе на постоянном токе
}

\author{
(C) А.Г. Веселов, ${ }^{1}$ В.И. Елманов, ${ }^{1}$ О.А. Кирясова, ${ }^{1}$ Ю.В. Никулин ${ }^{1,2}$ \\ ${ }^{1}$ Саратовский фрилиал института радиотехники и электроники им. В.А. Котельникова РАН, \\ 410019 Саратов, Россия \\ ${ }^{2}$ Саратовский национальный исследовательский государственный университет им. Н.Г. Чернышевского, \\ 410012 Саратов, Россия \\ e-mail: labsftwo@mail.ru
}

Поступило в Редакцию 23 июля 2018 г.

В окончательной редакции 23 июля 2018 г.

Принято к публикации 28 ноября 2018 г.

Для пленок $\mathrm{ZnO}$ толщиной $\sim 0.45-1.2 \mu \mathrm{m}$, синтезируемых в планарной магнетронной системе на постоянном токе в сдвиговой геометрии осаждения, исследована зависимость угла наклона текстуры и эффективности возбуждения сдвиговых волн от давления рабочего газа в диапазоне 0.14-0.74 mTorr, при котором происходит переход режима осаждения из бесстолкновительного в близкий к диффузному. Показано, что увеличение давления с $\sim 0.14-0.24$ до 0.74 mTorr приводит к уменьшению угла наклона столбчатой текстуры с $\sim 25-27$ до $\sim 7^{\circ}$ и снижению эффективности возбуждения звука. Пленки, синтезированные при давлениях $\sim 0.14-0.24 \mathrm{mTorr}$ близких к границе перехода разряда от таунсендсовского к тлеющему, характеризуются наилучшей эффективностью возбуждения сдвиговых волн - наблюдается наименьшая величина вносимых потерь при толщинах $0.45-0.75 \mu \mathrm{m}$, число наблюдаемых эхо-импульсов достигает 20-40, что позволяет наблюдать отраженный звук с задержкой до $80 \mu$ s при длине кристаллазвукопровода $10 \mathrm{~mm}$.

DOI: 10.21883/JTF.2019.05.47484.289-18

\section{Введение}

Широкий спектр применения пьезоактивных текстурированных пленок $\mathrm{ZnO}$ в СВЧ-устройствах акустои оптоэлектроники (резонаторы, фильтры, оптические модуляторы, линии задержки, конвольверы) [1-15], а также их устойчивость к физическому воздействию (вибрация, температура, электромагнитное излучение) являются причиной неослабевающего интереса к разработке технологий синтеза пьезоактивных текстурированных пленок $\mathrm{ZnO}$ толщиной менее $1 \mu \mathrm{m}$ и исследованию их свойств.

C точки зрения применения текстурированных пленок $\mathrm{ZnO}$ в области акустоэлектроники особый интерес представляют пленки с наклонной или лежащей текстурой (ось $C$ направлена под углом или параллельно плоскости пленки $\mathrm{ZnO}$ ). Это обусловлено рядом отличительных особенностей, которыми обладают такие пленки по сравнению с пленками с прямой текстурой (ось $C$ направлена перпендикулярно поверхности пленки $\mathrm{ZnO})$. В частности, акустические резонаторы на объемных сдвиговых волнах имеют наполовину меньшие температурный коэффициент частоты и площадь электродов, чем у резонаторов на продольных волнах, что способствует уменьшению массо-габаритов устройств на их основе. Кроме того, сдвиговые волны обладают почти вдвое меньшей скоростью распространения по сравнению с продольными волнами, что позволяет создавать линии задержки (ЛЗ) с более короткими звукопроводами при том же времени задержки сигнала по сравнению с ЛЗ на продольных волнах. Отметим, что интерес к развитию технологий формирования и исследованию свойств пьезоактивных пленок $\mathrm{ZnO}$ с наклонной текстурой дополняет способность возбуждения такими пленками поверхностных горизонтальных (SH) акустических волн, а также возможность их применения в интегральной оптоэлектронике и создания акусто-оптических приборов спектрального анализа на основе коллинеарной дифракции на объемных сдвиговых волнах.

На сегодняшний день для синтеза пьезоактивных пленок $\mathrm{ZnO}$ с наклонной или лежащей текстурой наиболее часто применяются методы магнетронного распыления (МР) - высокочастотного (ВЧ) [2,3,6,9,16-22] и на постоянном токе (МРТП) [23-26], а также реактивное $[1,2,5]$, электронно-лучевое [27] и ионно-лучевое [28] распыления. Для методов магнетронного распыления наибольшее влияние на пьезоакустические и структурные свойства формируемых пленок $\mathrm{ZnO}$ оказывают давление $P$ и состав рабочего газа $[5,16,17,19,22]$, температура подложки $T_{s}[5,16,17,22,24]$, расстояние между мишенью и подложкой [22], мощность магнетронного разряда $E$ и скорость осаждения $v$ [16]. При этом, как было показано ранее, для получения высококачественных пленок $\mathrm{ZnO}$ с наклонной или лежащей текстурой методом MP осаждение пленок $\mathrm{ZnO}$ следует производить при низком давлении рабочего газа 
$P<7$ mTorr $[1,2,19-21,24-26]$ и как можно меньшем расстоянии $L$ между распыляемой мишенью и подложкой $[1,22]$. Выбор низкого $P$ и малого $L$ обусловлен стремлением обеспечить режим пролета атомов распыляемой мишени близкий к бесстолкновительному [29], при котором формируются пленки с лучшими структурными характеристиками (плотность, химическая чистота, кристалличность) и адгезией, по сравнению с пленками, осаждаемыми при высоком давлении ( $P>7$ mTorr), когда режим пролета атомов близок к диффузному. Кроме того, диффузный режим осаждения, если не созданы какие-либо специальные условия [18], способствует формированию в пленках кристаллической ориентации с минимальной поверхностной энергией прямая текстура (0002) для $\mathrm{ZnO}[17,19]$.

Отметим, что при формировании пленок $\mathrm{ZnO}$ с наклонной текстурой независимо от выбора параметров $P, T_{s}, E$ и $v$, особое значение приобретает необходимость реализации ориентирующего фактора, определяющего наклон текстуры пленки. Среди ориентирующих факторов, применяемых в технологии синтеза пленок ZnO с наклонной текстурой, наиболее распространенным является наклон подложки относительно плоскости магнетрона [2,3,19,20-22,26-28]. Однако наклон подложки способствует формированию пленок с высокой неоднородностью толщины и угла наклона кристаллитов [26] по всей площади пленки, что приводит к искажению фронта распространения возбужденной в кристалле-звукопроводе акустической волны и ограничивает область применения таких пленок. К ориентирующим факторам, лишенным этого недостатка, можно отнести сдвиговую геометрию осаждения [17,19,24,25], при которой подложка располагается над зоной эрозии мишени без наклона относительно плоскости распыляемой мишени, а также использование различных ориентирующих подслоев $[2,22]$, ионной бомбардировки $[18,27]$ и напряжения смещения на подложке [18].

Для методов магнетронного распыления (ВЧ и на постоянном токе) с оптимизированными ростовыми режимами и способами ориентации оси текстуры была показана возможность формирования пьезоактивных пленок $\mathrm{ZnO}$ с наклонной или лежащей текстурой толщиной $0.8-10 \mu \mathrm{m}[2,3,6,17-21,24,25,28]$. Возможность создания пьезоактивных пленок $\mathrm{ZnO}$ толщиной менее $0.8 \mu \mathrm{m} \mathrm{c}$ однородной по всей площади подложки-звукопровода наклонной текстурой, представляющих наибольший интерес с точки зрения применения в устройствах акустоэлектроники СВЧ-диапазона, обсуждалась в работе [25]. Так, для пленок $\mathrm{ZnO}$, осаждаемых при $P \approx 0.26$ mTorr методом МРПТ в сдвиговой геометрии, с помощью химического травления в водном растворе $\mathrm{HCl}$, было показано, что получаемые пленки $\mathrm{ZnO}$ с наклонной текстурой толщиной $\sim 0.9 \mu \mathrm{m}$ сохраняют пьезоактивность и способны возбуждать сдвиговые волны вплоть до толщин $\sim 0.4 \mu \mathrm{m}$.

Отметим, что для сдвиговой геометрии осаждения характерно наличие касательной составляющей скорости движения атомов распыляемой мишени к плоскости подложки, величина которой может зависеть от давления рабочего газа $P$. Следует ожидать, что чем выше давление $P$, тем меньше будет касательная составляющая скорости за счет возросшего рассеяния атомов распыляемого материала мишени на атомах рабочего газа при переходе режима осаждения из бесстолкновительного в диффузный. Для пленок $\mathrm{ZnO}$, синтезируемых методом ВЧ-магнетронного распыления в сдвиговой геометрии, влияние давления рабочего газа на текстуру пленок исследовалось в $[17,19]$. Однако для пленок $\mathrm{ZnO}$ с наклонной текстурой, синтезируемых методом МРПТ в сдвиговой геометрии [24,25], вопрос о влиянии давления рабочего газа на эффективность возбуждения сдвиговых волн и микроструктуру пленок не обсуждался.

В настоящей работе для пленок $\mathrm{ZnO}$ с наклонной текстурой, осаждаемых методом МРПТ в сдвиговой геометрии, определен диапазон давлений рабочего газа $P$ и времени осаждения $t$, при которых происходит стабильный синтез пьезоактивных пленок $\mathrm{ZnO}$ толщиной $d \approx 0.45-1.2 \mu \mathrm{m}$. Показано, что синтез пленок $\mathrm{ZnO}$ с наклонной текстурой возможен в достаточно широком диапазоне давлений рабочего газа $P \approx 0.14-0.74$ mTorr, однако наименьшие значения вносимых потерь и наилучшая эффективность возбуждения сдвиговых волн (по величине вносимых пленкой потерь и числу наблюдаемых эхо-импульсов) достигаются для пленок, осаждаемых при давлениях рабочего газа вблизи границы зажигания плазмы $P \approx 0.14-0.24$ mTorr (переход разряда от таунсендсовского к тлеющему).

\section{Эксперимент}

Синтез пленок $\mathrm{ZnO}$ толщиной $d \approx 0.45-1.2 \mu \mathrm{m}$ производился в планарной несбалансированной магнетронной распылительной системе на постоянном токе с квазизамкнутым объемом зоны горения плазмы (рис. 1). Квазизамкнутый объем представлял собой кварцевый стакан диаметром $D \approx 40 \mathrm{~mm}$ и высотой $h \approx 23 \mathrm{~mm}$, нижняя часть которого ограничивалась магнитной системой магнетрона (катод), а верхняя - пластиной из алюминия (анод, находящийся под потенциалом „земли“) с отверстием диаметром $8 \mathrm{~mm}$ над зоной эрозии мишени. Базовое давление в ростовой камере составляло $P_{b} \approx 0.01$ mTorr. В качестве рабочего газа использовалась смесь газов - 40\% кислорода (чистота $99.999 \%$ ) и $60 \%$ аргона (чистота 99.999\%). В качестве мишени использовался диск $\mathrm{Zn}$ (диаметр $\sim 40 \mathrm{~mm}$, толщина $\sim 1 \mathrm{~mm}$, чистота 99.99\%). Осаждение пленок $\mathrm{ZnO}$ производилось при температуре подложки $T_{s} \approx 300^{\circ} \mathrm{C}$ и давлениях рабочего газа вне квазизамкнутого объема $P \approx 0.14,0.24,0.34,0.44,0.54$ и 0.74 mTorr. Давление $P_{i v}$ внутри квазизамкнутого объема на $\sim 0.6$ mTorr превышало $P$ (далее по тексту и на рисунках приводится давление $P$ вне квазизамкнутого объема). Измерение давления рабочего газа в процессе напыления пленки 


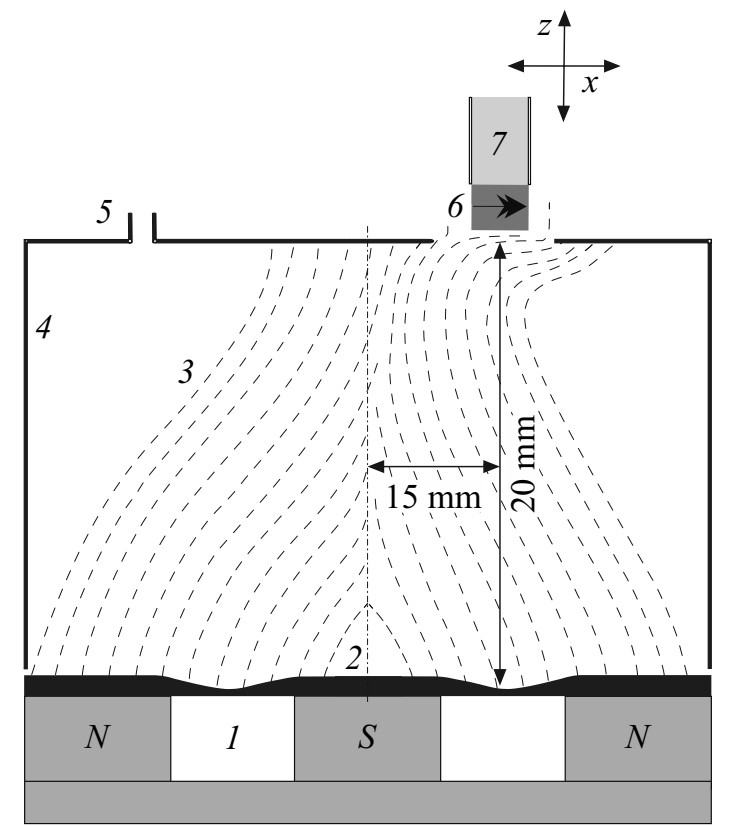

Pис. 1. Схема квазизамкнутого объема для осаждения пленок $\mathrm{ZnO}$ с наклонной текстурой: 1 - магнитная система магнетрона, 2 - мишень $\mathrm{Zn}, 3$ - зона горения плазмы (штриховыми линиями схематически показана светящаяся область рекомбинационного горения), 4 - квазизамкнутый объем с анодом из алюминия и отверстием для размещения подложкизвукопровода, 5 - место напуска газа в квазизамкнутый объем, 6 - пленка $\mathrm{ZnO}, 7$ - подложка-звукопровод АИГ с нагревателем, положение которой юстировалось над зоной эрозии мишени за счет перемещения по осям $X$ и $Z$.

осуществлялось с помощью ионизационного вакуумметра ВИТ-2. В процессе напыления на мишень подавалось постоянное напряжение величиной $U \approx-500 \mathrm{~V}$, при этом ток разряда поддерживался на уровне $I \approx 120 \mathrm{~mA}$ для всех $P$. Мощность магнетронного разряда $E$ и скорость осаждения $\mathrm{v}$ зависели от давления $P$ линейно и составляли $E \approx 41-33 \mathrm{~W}$ и $v \approx 40-20 \mathrm{~nm} / \mathrm{min}$ для $P \approx 0.14-0.74$ mTorr. В качестве подложки-звукопровода использовался монокристаллический алюмоиттриевый гранат, легированный лютецием (АИГ) с площадью поверхности под осаждение пленки $4 \times 4 \mathrm{~mm}$ и длиной $10 \mathrm{~mm}$.

Для создании пьезопреобразователей на основе пленок $\mathrm{ZnO}$ в качестве материала подслоя использовался хром $(\mathrm{Cr})$ чистотой 99.99\%. Металлический подслой $\mathrm{Cr}$ толщиной $400 \mathrm{~nm}$ осаждался на подложку АИГ при температуре $T_{s} \approx 300^{\circ} \mathrm{C}$ термическим осаждением из танталового тигля при давлении остаточной атмосферы $P_{b} \approx 0.05$ mTorr. После нанесения подслоя $\mathrm{Cr}$ подложка без развакууммирования размещалась по центру отверстия в аноде квазизамкнутого объема над зоной эрозии мишени на расстоянии $L \approx 23 \mathrm{~mm}$ от поверхности мишени, где в течение времени $t \approx 12-40 \min$ при $T_{s} \approx 300^{\circ} \mathrm{C}$ происходило осаждение пленок $\mathrm{ZnO}$. Осаждение пленок $\mathrm{ZnO}$ осуществлялось на один и тот же кристалл АИГ, для этого после каждого напыления пленки $\mathrm{ZnO}$ и ее исследования она удалялась путем химического травления в водном растворе кислоты $\mathrm{HCl}$. Отметим, что пленки $\mathrm{ZnO}$ с наклонной текстурой, выращиваемые по описанной в настоящей работе технологии, характеризуются высокой прочностью и адгезией к подложке - удалить пленку $\mathrm{ZnO}$ с подложки АИГ было возможно только путем химического травления в азотной или соляной кислоте.

В настоящей работе исследовались пьезоакустические свойства и микроструктура для двух серий пленок: серия № 1 - пленки $\mathrm{ZnO}$ осаждались при давлениях $P \approx 0.14-0.74$ mTorr в течение $t \approx 20 \mathrm{~min} \mathrm{с} \mathrm{целью}$ определения влияния давления рабочего газа на толщину пленки и эффективность возбуждения звука; серия № $2-$ пленки $\mathrm{ZnO}$ осаждались при $P \approx 0.14$ mTorr $(t \approx 12-30 \mathrm{~min}), \quad P \approx 0.44 \mathrm{mTorr} \quad(t \approx 20$ и $30 \mathrm{~min})$, $P \approx 0.54$ mTorr $(t \approx 20$ и $30 \mathrm{~min}), P \approx 0.74$ mTorr $(t \approx 30$ и $40 \mathrm{~min}$ ) с целью определения различий в эффективности возбуждения звука и наклоне текстуры для пленок с близкой по значению толщиной, выращенных при разных $P$. Изменение $t$ позволяло формировать пленки $\mathrm{ZnO}$ различной толщины, при этом увеличение времени осаждения до 30-40 min для высоких давлений рабочего газа $(P \approx 0.54-0.74$ mTorr $)$ способствовало увеличению толщины $d$ формируемых пленок до $750 \mathrm{~nm}$.

Возбуждение и регистрация акустических колебаний в пьезопреобразователях на основе текстурированных пленок $\mathrm{ZnO}$ производились на измерительной установке, состоящей из импульсного СВЧ-генератора $(845 \mathrm{MHz}$, длительность импульса $1-5 \mu \mathrm{s})$, генератора синхроимпульсов, усилителя и осциллографа. СВЧ-импульсы мощностью $1 \mu \mathrm{W}-0.5 \mathrm{~W}$ подводились непосредственно к пьезоактивному преобразователю через СВЧ-зонд с центральным электродом диаметром $100 \mu \mathrm{m}$, что позволяло оценивать однородность текстуры пленки с шагом $\sim 100 \mu \mathrm{m}$ по всей поверхности подложки-звукопровода. В эксперименте измерялись общие потери звука в пленке и подложке-звукопроводе. Отметим, что за счет отсутствия согласования подводящей зондовой СВЧ-линии с пленкой $\mathrm{ZnO}$, а также различия акустических импедансов подложки-звукопровода АИГ и пленки $\mathrm{ZnO}$, наименьшая величина потерь, наблюдаемая нами в эксперименте, составляла около $47 \mathrm{~dB}$. Для определения качества выращенной пленки $\mathrm{ZnO}$ с точки зрения эффективности возбуждения звука, наряду с потерями оценивалось число $N$ эхо-импульсов, наблюдаемых на осциллограммах. Если на осциллограммах наблюдалось $N \approx 20-40$ эхо-импульсов и их число было постоянным (в пределах $\pm 1-2$ ) на всей площади пленки, то считалось, что пленка $\mathrm{ZnO}$ обладает однородной по площади подложки текстурой и толщиной и эффективно возбуждает звук.

Исследование поперечного сечения пленок $\mathrm{ZnO}$ производилось методом сканирующей электронной микроскопии (СЭМ) (Auriga, CarlZeiss). Оценка толщины пленок $d$ и ее однородности по площади подложки производилась для каждого образца методом профилометрии (Dectak 150, Veeco) с точностью 2-3\%. 

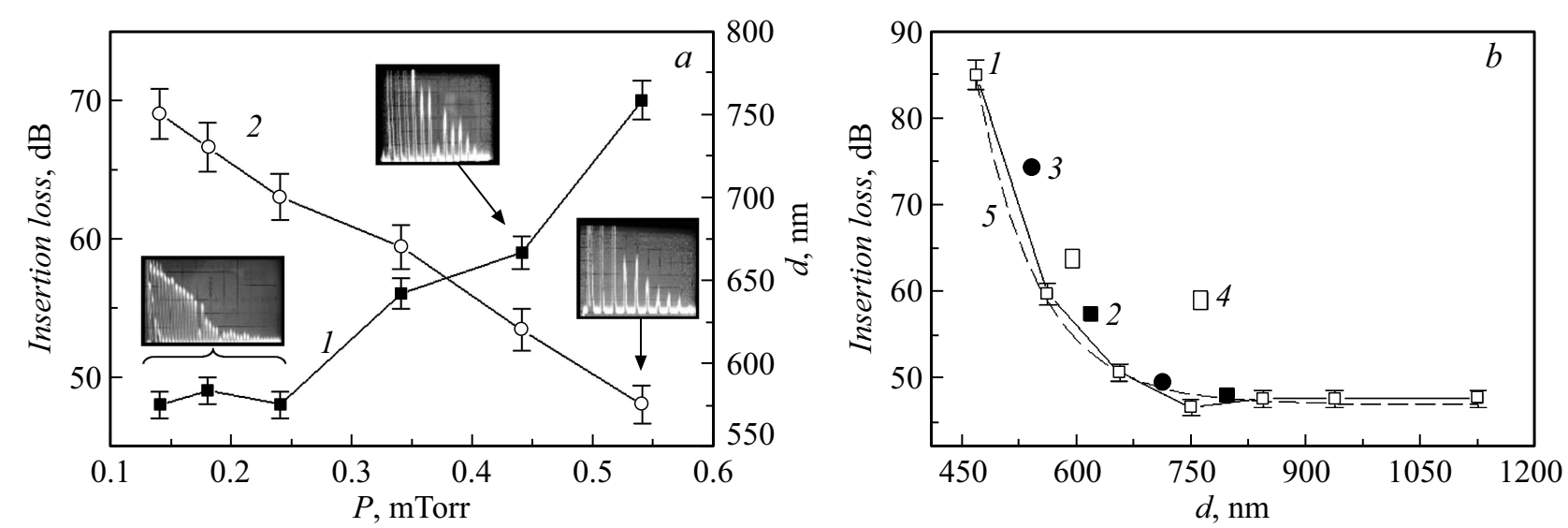

Рис. 2. $a$ - зависимости величины вносимых пленкой потерь $(1)$ и толщины пленки $d(2)$ от давления рабочего газа $P$. На вставках к рисунку приведены типичные осциллограммы эхо-импульсов, наблюдаемые для пленок, синтезируемых при $P \approx 0.14-0.24$ mТorr (масштаб осциллограммы $-4 \mu \mathrm{s} / \mathrm{cm}), P \approx 0.44$ mTorr (масштаб осциллограммы $-1.5 \mu \mathrm{s} / \mathrm{cm}$ ) и $P \approx 0.54 \mathrm{mTorr}$ (масштаб осциллограммы $-1 \mu \mathrm{s} / \mathrm{cm})$. Величина погрешности для кривых $1,2-2 \% ; b-$ зависимости величины вносимых пленкой потерь от толщины пленки $d$ для пленок $\mathrm{ZnO}$, выращенных при $P \approx 0.24$ (кривая 1$), \approx 0.44(2), \approx 0.54(3)$ и $\approx 0.74$ mTorr $(4)$. Штриховой линией 5 показана экспоненциальная аппроксимация кривой 1 . Величина погрешности для кривой $1-2 \%$.

\section{Результаты и обсуждение}

Результаты исследования пьезоакустических свойств выращенных пленок $\mathrm{ZnO}$ толщиной $d \approx 450-1200 \mathrm{~nm}$ показали, что во всем диапазоне давлений рабочего газа $P \approx 0.14-0.74$ mTorr формируются пьезоактивные пленки $\mathrm{ZnO}$ с наклонной текстурой, при этом созданные на их основе пьезоакустические преобразователи способны эффективно возбуждать сдвиговые волны при толщинах пленки менее $0.7 \mu \mathrm{m}$. На рис. 2, $a$ приведены зависимости величины вносимых потерь (кривая 1) и толщины пленки $d$ (кривая 2) от $P$ для пленок $\mathrm{ZnO}$, синтезируемых в течение $t \approx 20 \min$ (серия № 1 ). На вставках к рис. 2 приведены типичные осциллограммы эхо-импульсов, наблюдаемые для пленок, синтезируемых при $P \approx 0.14-0.24, \approx 0.44$ и $\approx 0.54$ mTorr. Из рисунка видно, что увеличение $P$ с 0.14 до 0.54 mTorr приводит к уменьшению толщины пленок с 750 до $570 \mathrm{~nm}$ и росту вносимых пленкой потерь с 47 до $70 \mathrm{~dB}$. Кроме того, с ростом $P$ наблюдается снижение эффективности возбуждения звука, которое проявляется в уменьшении числа $N$ наблюдаемых эхо-импульсов с $\sim 40$ до $\sim 10-13$ (см. вставки на рис. 2, $a$ ). Наблюдаемое уменьшение толщины пленки $d$ с ростом давления $P$ связано со снижением скорости роста с $v \approx 40$ до $v \approx 20 \mathrm{~nm} / \mathrm{min}$ при переходе из бесстолкновительного в близкий к диффузионному режим за счет возрастающего рассеяния атомов распыляемой мишени на атомах рабочего газа, тогда как уменьшение числа эхо-импульсов вызвано изменением микроструктуры пленки и ухудшением однородности текстуры по площади пленки.

Для определения различий в эффективности возбуждения звука и наклоне текстуры для пленок $\mathrm{ZnO}$ с близкой по значению толщиной, но выращенных при разных $P$, было проведено сравнение пьезоакустиче- ских свойств и микроструктуры пленок серии № 2. На рис. 2, $b$ приведена зависимость величины вносимых потерь от толщины $d$ для пленок $\mathrm{ZnO}$, синтезируемых при $P \approx 0.24$ mTorr (кривая 1 ) в течение $t \approx 12-30 \mathrm{~min}$. Из рисунка видно, что зависимость величины потерь от толщины пленки $d$ близка к экспоненциальной (экспоненциальная аппроксимация кривой 1 показана штриховой линией 5 на рис. 2,b). Отдельными точками рядом с кривой 1 обозначены значения вносимых потерь для пленок, выращенных при $P \approx 0.44(2), \approx 0.54(3)$ и $\approx 0.74$ mTorr $(4)$ в течение $t \approx 20,30$ и $40 \mathrm{~min}$. Сравнение данных показывает, что в пленках толщиной $d \leq 600 \mathrm{~nm}$ наименьшими потерями обладают пленки, осажденные при низком давлении рабочего газа $P \approx 0.24$ mTorr (рис. 2, $b$, кривая 1 ). В пленках толщиной $d>700 \mathrm{~nm}$, выращенных при $P \approx 0.24-0.54$ mTorr, величина потерь почти не зависит от $P$ и составляет $47-50 \mathrm{~dB}$, тогда как для пленок, осажденных при $P \approx 0.74$ mTorr, величина потерь оказывается на $12 \mathrm{~dB}$ больше $(\sim 60 \mathrm{~dB})$, чем у пленок при $P \approx 0.54-0.24$ mTorr.

Наблюдаемое изменение величины потерь и эффективности возбуждения звука пленками $\mathrm{ZnO}$ с ростом $P$ связано со структурными различиями пленок, осажденных при высоких $(P \approx 0.54$ mTorr $)$ и низких $(P \approx 0.14-0.24$ mTorr $)$ давлениях рабочего газа, а именно с различием в угле наклона $\alpha$ столбчатой структуры относительно нормали к пленке. На рис. 3 приведены типичные изображения сколов и осциллограммы пленок $\mathrm{ZnO}$, осажденных при $P \approx 0.24,0.54$ и 0.74 mTorr. Видно, что при всех $P$ пленки характеризуются столбчатой структурой, угол наклона $\alpha$ которой линейно зависит от $P$ и уменьшается с $\alpha \approx 25^{\circ}$ при $P \approx 0.24$ mTorr до $\alpha \approx 7^{\circ}$ при $P \approx 0.74$ mTorr. Уменьшение угла наклона $\alpha$ приводит к тому, что эффективность возбуждения сдви- 

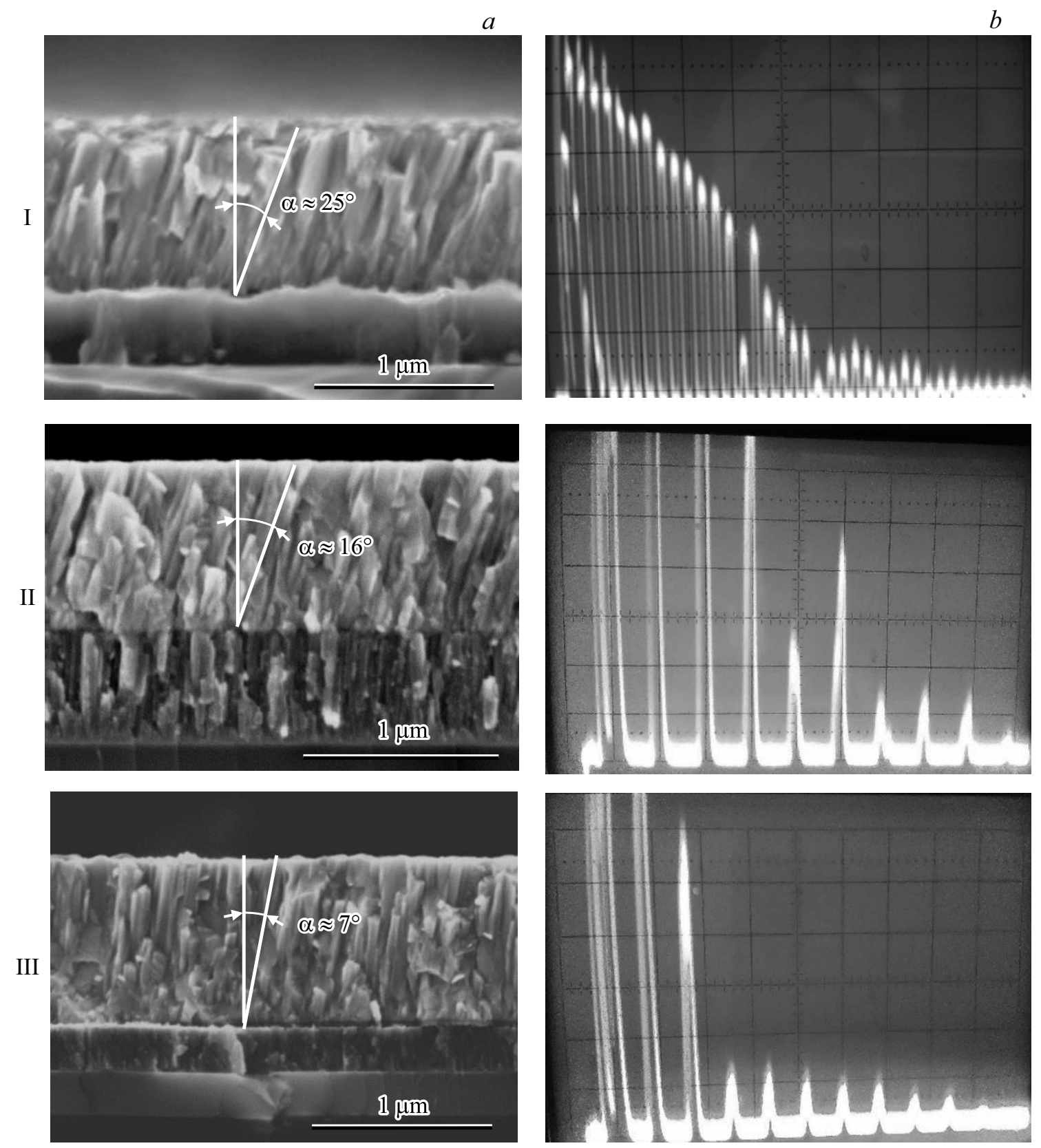

Рис. 3. СЭМ-изображения сколов $(a)$ и осциллограммы сдвиговых эхо-импульсов $(b)$ для пленок $\mathrm{ZnO}$ с наклонной текстурой, выращенных при $P \approx 0.24$ mTorr $(\mathrm{I}), P \approx 0.54$ mTorr (II) и $P \approx 0.74$ mTorr (III). Масштаб для осциллограмм: I -4 , II -1 , III $1 \mu \mathrm{s} / \mathrm{cm}$.

говых волн снижается с ростом $P$. Так, в пленке толщиной $d \approx 750 \mathrm{~nm}$, выращенной при $P \approx 0.24$ mTorr, на осциллограмме наблюдается однородная по поверхности пленки сетка из $\sim 40$ эхо-импульсов (рис. 3, $a$ ), при этом потери между соседними эхо-импульсами по порядку величины равны потерям на один проход в кристаллезвукопроводе. Для пленки такой же толщины, выращенной при $P \approx 0.54-0.74$ mTorr, сетка состоит не более чем из 10-13 эхо-импульсов (рис. $3, b, c$ ) и является достаточно неоднородной по площади пленки. В нашем случае под неоднородностью сетки эхо-импульсов мы подразумеваем различие числа $N$ эхо-импульсов, наблюдаемых на разных участках пленки. Как правило, для пленок, выращенных при $P \approx 0.74$ mTorr, разброс $N$ составлял 4-5 эхо-импульсов, тогда как для пленок, осаждаемых при $P \approx 0.14-0.24$ mTorr, разброс не превышал 1-2 эхо-импульса.

Полученные результаты позволяют с учетом зависимости $\alpha(P)$, близкой к линейной (рис. 4), оценить давление рабочего газа $P$ и угол $\alpha$, при которых пленки $\mathrm{ZnO}$ будут проявлять максимальную и минимальную эффективность возбуждения сдвиговых волн. 


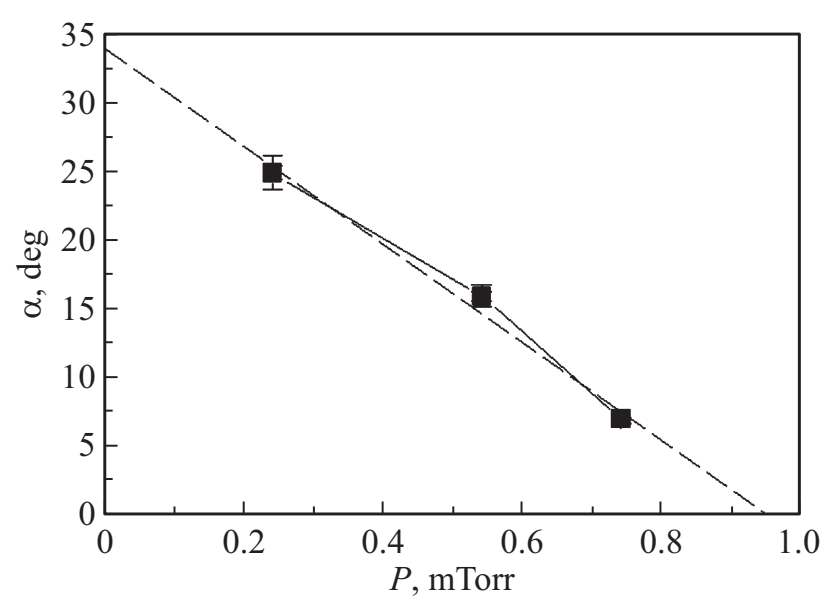

Pис. 4. Зависимость угла наклона столбчатой текстуры от давления рабочего газа $P$. Штриховой линией обозначена среднеквадратичная аппроксимация зависимости $\alpha(P)$. Величина погрешности измерения угла $\alpha-5 \%$.

Из линейной аппроксимации, представленной на рис. 4, следует, что давление, при котором угол наклона $\alpha \approx 0$ (минимальная эффективность возбуждения звука), соответствует $P \approx 0.95$ mTorr, а наименьшее давление рабочего газа, соответствующее порогу зажигания плазмы $(P \approx 0.1-0.12 \mathrm{mTorr})$, дает значение угла наклона столбчатой структуры $\alpha \approx 29-30^{\circ}$ (наибольшая эффективность возбуждения сдвиговых волн).

\section{Заключение}

Для пленок $\mathrm{ZnO}$ с наклонной текстурой, осаждаемых методом МРПТ в сдвиговой геометрии, установлено существенное влияние давления рабочего газа $(P \approx 0.14-0.74$ mTorr $)$ на угол наклона $\alpha$ текстуры пленки, толщину пленки $d$ и эффективность возбуждения звука. Показано, что эффективность возбуждения сдвиговых волн, определяемая по величине вносимых пленкой потерь и числу наблюдаемых эхо-импульсов, снижается с ростом $P$ за счет уменьшения угла наклона текстуры пленки с $\alpha \approx 25-27^{\circ}$ при $P \approx 0.24-0.14$ mTorr до $\alpha \approx 7^{\circ}$ при $P \approx 0.74$ mTorr. Причиной изменения угла наклона текстуры в зависимости от давления рабочего газа является переход режима осаждения от бесстолкновительного к диффузному, при котором имеет место существенное рассеяние атомов распыляемого материала мишени на атомах рабочего газа и соответственно изменяется угол их подлета к подложке. Наиболее эффективно возбуждают звук пленки $\mathrm{ZnO}$, синтезированные при $P$, близком к порогу зажигания плазмы $P \approx 0.14-0.24$ mTorr. Получаемые при этих $P$ пленки $\mathrm{ZnO}$ толщиной $d \approx 0.45-0.75 \mu \mathrm{m}$ имеют наибольший угол наклона кристаллитов $\alpha \approx 25-27^{\circ}$, характеризуются однородной текстурой на всей площади пленки и позволяют наблюдать отраженный звук c задержкой до $80 \mu \mathrm{s}$ при длине кристалла-звукопровода $1 \mathrm{~cm}$.

Полученные в работе результаты могут представлять интерес для развития технологии синтеза тонких $(d<0.5 \mu \mathrm{m})$ пьезоактивных пленок $\mathrm{ZnO}$ с наклонной текстурой, перспективных при разработке акустоэлектронной элементной базы устройств обработки информации СВЧ-диапазона.

Работа выполнена при финансовой поддержке РФФИ, проекты № 16-37-60052, 16-29-14058.

\section{Список литературы}

[1] Rughoobur G., De Miguel-Ramos M., Mirea T., Clement M., Olivares J., Díaz-Durán B., Sangrador J., Miele I., Milne W.I., Iborra E., Flewitt A.J. // Appl. Phys. Lett. 2016. Vol. 108. P. 034103-1-5. DOI: 10.1063/1.4940683

[2] Rughoobur G., García-Gancedo L., Flewitt A.J., Milne W.I., De Miguel-Ramos M., Clement M., Mirea T., Olivares J., Iborra E. // Europ. Frequency and Time Forum (EFTF). 2014. P. 297-300. DOI:10.1109/EFTF.2014.7331491

[3] Yoshino Y. // J. Appl. Phys. 2009. Vol. 105. P. 061623-1-7. DOI: $10.1063 / 1.3072691$

[4] Prasad M., Sahula V., Khanna V.K. // IEEE Trans. On Device and Mat. Reliability. 2014. Vol. 14. N 1. P. 545-553. DOI: 10.1109/TDMR.2013.2271245

[5] Link M., Schreiter M., Weber J., Gabl R., Pitzer D., Primig R., Wersing W., Assouar M.B., Elmazria O. // J. Vac. Sci. Technol. A. 2006. Vol. 24. P. 218-220. DOI: $10.1116 / 1.2165658$

[6] Nalamwar A.L., Wagers R.S., Epstein M. // J. Appl. Phys. 1977. Vol. 48. N 6. P. 2175-2178. DOI: $10.1063 / 1.324017$

[7] Yan Z., Zhou X.Y., Pang G.K.H., Zhang T., Liu W.L., Cheng J.G., Song Z.T., Feng S.L., Lai L.H., Chen J.Z., Wang Y. // Appl. Phys. Lett. 2007. Vol. 90. P. 143503-1-3. DOI: $10.1063 / 1.2719149$

[8] Qin L., Chen Q., Cheng H., Chen Q., Li J.-F., Wang Q.-M. // J. Appl. Phys. 2011. Vol. 110. P. 094511-1-11. DOI: $10.1063 / 1.3657781$

[9] Dwivedi M., Bhargava J., Sharma A., Vimal V., Eranna G. // IEEE Sensors Journ. 2014. Vol. 14. N 5. P. 1577-1582. DOI: $10.1109 /$ JSEN.2014.2298879

[10] Алексеев С.Г., Гуляев Ю.В., Котелянский И.М., Мансфельд Г.Д. // УФН. 2005. Т. 175. № 8. С. 895-900.

[11] Hickernell F.S. // ТИИЭР. 1976. Т. 64. № 5. С. 70-76.

[12] Coldren L.A. // ТИИЭР. 1976. Т. 64. № 5. С. 246-249.

[13] Özgür Ü., Alivov Ya. I., Liu C., Teke A., Reshchikov M.A., Doğan S., Avrutin V., Cho S.-J., Morkoç H. // J. Appl. Phys. 2005. Vol. 98. P. 041301-1-103. DOI: $10.1063 / 1.1992666$

[14] Ching K.-L., Li G., Ho Y.-L., Kwok H.-S. // Cryst. Eng. Comm. 2016. Vol. 18. P. 779-786. DOI: $10.1039 / C 5 C E 02164 B$

[15] Djurisic A.B., Ng A.M.C., Chen X.Y. // Progress in Quantum Electronics. 2010. Vol. 34. P. 191-259. DOI:10.1016/j.pquantelec.2010.04.001

[16] Fujimura N., Nishihara T., Goto S., Xu J., Ito T. // J. Crystal Growth. 1993. Vol. 130. P. 269-279.

[17] Kawamoto T., Yanagitani T., Matsukawa M., Watanabe Y. // Japan. J. Appl. Phys. 2007. Vol. 46. N 7B. P. 4660-4664. DOI: $10.1143 / J J A P .46 .4660$ 
[18] Takayanagi S., Yanagitani T., Matsukawa M., Watanabe Y.// IEEE Intern. Ultrason. Sympos. Proceed. 2010. P. 1060-1063. DOI: 10.1109/ULTSYM.2010.5935655

[19] Takayanagi S., Yanagitani T., Matsukawa M., Watanabe Y.// IEEE Intern. Ultrason. Sympos. Proceed. 2011. P. 2317-2320. DOI: 10.1109/ULTSYM.2011.0575

[20] Yanagitani T., Mishima N., Matsukawa M., Watanabe Y. // IEEE Trans. Ultrason. Ferroelectrics and Frequency Control. 2007. Vol. 54. N 4. P. 701-704.

DOI: $10.1109 /$ TUFFC.2007.303

[21] Yanagitani T., Kiuchi M., Matsukawa M., Watanabe Y. // IEEE Trans. Ultrason. Ferroelectrics and Frequency Control. 2007. Vol. 54. N 8. P. $1680-1686$.

DOI: $10.1109 /$ TUFFC.2007.439

[22] Lehmann H.W., Widmer R. // J. Appl. Phys. 1973. Vol. 44. N 9. P. $3868-3879$. DOI: $10.1063 / 1.1662864$

[23] Zhao Z., Pan C., Gao C., Wang C. // IEEE Intern. Vacuum Electron. Conf. (IVEC). 2015 . P. 1-2.

DOI: 10.1109/IVEC.2015.7224023

[24] Веселов А.Г., Елманов В.И., Кирясова О.А., Никулин Ю.В. // ЖТФ. 2017. Т. 87. Вып. 3. С. 448-452.

DOI: 10.21883/JTF.2017.03.44253.1849

[Veselov A.G., Elmanov V.I., Kiryasova O.A., Nikulin Yu.V. // Tech. Phys. 2017. Vol. 62. N 3. P. 470-474.

DOI: $10.1134 / \mathrm{S} 1063784217030264]$

[25] Веселов А.Г., Елманов В.И., Кирясова О.А., Никулин Ю.В. // ЖТФ. 2018. Т. 88. Вып. 1. С. 98-106.

DOI: 10.21883/JTF.2019.05.47484.289-18

[Veselov A.G., Elmanov V.I., Kiryasova O.A., Nikulin Yu.V. // Tech. Phys. 2018. Vol. 63. N 1. P. 95-103.

DOI: $10.1134 / \mathrm{S} 1063784218010279]$

[26] Minakata M., Chubachi N., Kikichi Y. // Japan. J. Phys. 1973. Vol. 12. N 3. P. 474-475.

[27] Yanagitani T., Kiuchi M. // J. Appl. Phys. 2007. Vol. 102. P. 044115-1-6. DOI: $10.1063 / 1.2772589$

[28] Yanagitani T., Kiuchi M. // IEEE Ultrason. Sympos. 2007. P. $1413-1416$.

[29] Somekh R.E. // J. Vac. Sci. Tech. A. 1984. Vol. 2. P. $1285-1291$. DOI: $10.1116 / 1.572396$ 